\title{
The Dilemma and Solutions of the Legal Regulation on the Card Drawing Mechanism in Games
}

\author{
Chenxi $\mathrm{Wu}^{1, *}$ \\ ${ }^{1}$ Wuhan University of Technology, Wuhan, Hubei, China \\ *Corresponding author. Email: 1262206766@qq.com

\begin{abstract}
With the rapid development of the game industry in China, the types of gameplay are becoming more and more diversified. Card drawing is a new rule of the game. Players spend money to draw props, but since the process is random, they don't necessarily get the prop with highest value. Under the stimulation of this mechanism, players can adopt the gambler's mentality to a certain degree. This paper mainly analyzes the similarities and differences between card drawing and gambling, and puts forward the regulation suggestions of the card drawing mechanism so that it can get rid of the suspected nature of gambling while keeping the playability, and points out the shortcomings and room for improvement of the card drawing mechanism, so that the game industry can grow healthily and contribute to the development of China's economy.
\end{abstract}

Keywords: card drawing, gambling, probability, big data

\section{INTRODUCTION}

With the development of the Internet, electronic games have become an important mode of life for people in terms of leisure and entertainment. The card drawing mechanism is adopted in some of the games that are popular today. Players can get props through this mechanism. They can get 10 different props randomly when they draw ten times but the levels and types of them cannot be guaranteed. The probability of drawing some valuable and rare props are extremely low and players may need to draw many times to get them. Yet the extra chances need to be bought by money. As a result, some players may spend a tremendous amount of money to get a prop. These card games are a bit like gambling. They all depend on luck and are addictive. In this process, the players will even have gambler's mentality and spend money in a rage. The system is controversial, with many players and the media linking it to gambling, which they see as a mechanism similar to gambling. In reality, this kind of card drawing system has become the core of many games. Some games are mainly profitable by the amount of money the players spend in the card drawing system. In many countries, players spend more money in this system than they can afford. However, at present, no country in the world has made laws or established legal precedents to regulate it in relevant aspects.

\section{OPERATION PRINCIPLE OF THE CARD DRAWING SYSTEM}

In electronic games, players generally have to complete tasks and collect cards in order to synthesize props. This method of acquiring props is a bit demanding as it requires a certain time and energy. In addition, there is a second way, which is to buy them directly with the currency in specific games which can generally be obtained by paying real yuan. These two ways are obviously exchange of equal values. Different from them both, the card drawing system features more randomness and depends on luck. A player may get a prop of high value with very low cost, and may also fail to get the one they want after spending a lot. This randomness and uncertainty increases the playability of the game and creates a thrill in the mind of players. Compared with equivalent exchange, this kind of card drawing mechanism can stimulate people to make impulse purchases. After not getting the props needed and paying a huge sunk cost, the player will stop at no expense until getting the card they want. With a certain social nature, current games form a certain community of players, in which comparison and peacock breed. The possession of high-value props will enable players to have a certain say in the community and be pursued by other players, which can satisfy their certain sense of superiority and vanity. And most games will have ranking and competition, in which the better the props are, the more points one can get, and thus higher ranking they will get. In this circumstance, players are stimulated to spend more money to draw cards. In previous games, drawing cards was just an auxiliary 
way to play. With the development of the times, in recent years, card drawing has even become the core of the game. In order to stimulate the card drawing, there even appear cards issued in limited time and number, the cards that can only be drawn in a certain time period or those that are really rare and won't be replenished after being sold out. Card drawing system has become the main means of profit for game manufacturers. "Azur Lane", "Shining Nikki", "Onmyoji", "fate" and other mobile games all stimulate players to draw cards though various means. For all the different props, the essence of them is the same.

\section{THE CARD DRAWING SYSTEM AND GAMBLING}

\section{A. Similarities between the card drawing system and gambling}

Gambling is betting a certain amount of money or something of value on something whose outcome is uncertain. It involves a price and an element of luck in order to obtain the expected reward. It is mainly to pay less to get more than the cost of profit, and in gambling luck is an important part. In this respect, the card drawing system is very similar to gambling. In order to get the chance to draw a card, one need to pay for the currency in the virtual game world, which is often purchased with the real money. So the virtual currency consumed is the equivalent of gambling. In the process of card drawing, luck counts a lot. Sometimes one can get a high-value prop in just one drawing, and sometimes they still can't get them after many drawing. This kind of drawing method with a certain probability is to rely on luck, and the rare props one finally get are the final reward. Under such operation of the card drawing system, the gambler's mentality can be stimulated in players, who will thus be so bewitched. Therefore, there is a certain similarity between the card drawing system and gambling.

\section{B. Difference between card drawing and gambling}

Gambling is banned in China. At the very beginning, the identification standard for gambling in games in China was for board games. Virtual currency in the game could not be converted into money in reverse. If the virtual currency in the game can directly be converted into RMB, the game would be regarded as gambling. Operators are not allowed to take a certain percentage of virtual currency after the gambling ends. There is a limit on the amount and number of bets placed in each game. If the amount and number of bets are not restricted, it will form gambling when beyond a certain limit.

The identification criteria of board games can also be a reference for the card drawing system. In the card drawing system, players draw the card not to obtain the actual monetary benefit, but to obtain the game props. These props are essentially virtual data, and the process is just a transaction between the player and the game's operator, in which money flows only one way. The sense of superiority and excitement that the gamer gets is also limited to the game, not the real world benefits as defined in gambling standard. But the game account can trade among the game players, but this kind of trade is only between players and is a way to deal with their virtual property. This kind of trade is also heavily dependent on the circle formed by the game itself, not like gambling, which directly trade money. It can be assumed that players buy virtual goods offered by game vendors though virtual currency. Virtual currencies are bought with real money, while virtual goods are made with time and money from game operators. Since the trade is directly between players and operators, there is no such a thing as a percentage of currency taken by operators. As long as game operators set certain limits on the number and times of card drawing, the practice is outside the scope of gambling. Moreover, most game operators set up a card drawing system in the game, mainly for the purpose of selling their own virtual goods by increasing the playability of the game, rather than making illegal profits through gambling mechanism. Although in some ways the card game is infinitely close to gambling, it cannot be regarded as gambling.

\section{The relationship between card drawing system and gambling}

There are both similarities and differences with gambling. It is very similar to gambling in stimulating people's psychology, requiring a certain price, relying on luck, and expecting certain benefits. But in the actual form it is different from gambling. As a game, the card drawing system has certain interests, which can increase the playability of the game. Moreover, the virtual wealth in the game cannot be directly converted into money. Moreover, the game involves a very limited number of people, and the transaction of virtual wealth has certain limitations. So the card drawing games is not equal to gambling. However, the probability and luck in the card drawing game cannot be regarded as a simple transaction. This mechanism needs to be controlled to a certain degree. Once the probability is too low, the player will spend money recklessly to draw the card, which is the same as in gambling. Generally speaking, the game of card drawing is on the edge of gambling. Once there is no supervision and regulation, it will develop in the direction of gambling. However, if the regulation is too absolute and strict, China's game industry will be hit hard and a large number of games in operation will be affected, to the detriment of the interests of the business. Therefore, it is necessary to define the boundary between card drawing and gambling. 


\section{The dilemma of the card drawing mechanism}

The card drawing mechanism has certain playability. Its randomness and uncertainty shows certain temptation to the player, can satisfy the players' demand to pursue stimulation. Therefore, even though there is a certain similarity between the card drawing mechanism and gambling, the card drawing mechanism is not prohibited by law. But once it is allowed to develop freely, it will always have the same bad consequences as gambling. The pursuit of rare props can be an incentive for players to play the game, but the overzealous pursuit of certain props can turn players into gamblers. Although the card drawing system and the traditional sense of gambling is not the same, they share the operating principle in essence. Therefore, it is important to set a clear boundary for the card drawing mechanism, so that it can guarantee playability while keeping it away from the harm of gambling.

\section{CHINA'S CURRENT RULES AND REGULATIONS ON CARD DRAWING MECHANISM AND THE DEFICIENCIES}

\section{A. Regulation on card drawing}

In 2019, China's game approval rules changed. Regulations were made on the number of times of card drawing per day, which defines an upper limit of the times every day. Players can make no more than 30 draws per time, make 10 draws continuously no more than 3 times, and no more than 50 times a day. In addition, the vendor must clearly indicate the number of remaining times for players. This is similar to the limit on the amount of each bet and the total sum in the gambling issue, preventing the players from overindulging, imposing restrictions on them, and stopping them from spending money endlessly. However, the fifty draws do not include the free number of times given away in the game. This rule guarantees the profits of the merchants and ensures that players who are too immersed in the game will not overspend because of the gambler's mentality.

The new rule also stipulates that the card drawing system will no longer display the probability, and it must be accurate to the number of draws to the point of which a prop can be drawn. The aim is to regulate the ceiling of card drawing game to prevent players from drawing too much cards and prevent the card drawing mechanism from becoming another form of gambling. A probability only, $10 \%$ for example, though indicating one win in every ten draws, is only a total probability that may not be true in an individual and one may fail to win once while others win at the first draw. It is friendlier to individual players that the rules be mad come. It is fairer for individual players that the probability of $10 \%$ be turned into one win in every ten draws and the average probability of all players be turned into a fixed probability. In this respect, the circumstance in which some unlucky player fail to win no matter how many times they try will be avoided, preventing them from entering a dead end. In addition, this can mitigate the gambler's mentality of players. When one really wants a prop, the guaranteed card drawing makes psychological preparations for them. It can be equated with equivalent exchange, preventing them from adopting the gambler's mentality.

These two new rules regulate the card drawing system from two aspects, and set a red line for the this game play, which weakens its similarity with gambling. But the core of the card drawing system and the player's stimulation still can't get rid of the gambling factor.

\section{B. The deficiencies of the regulation on card drawing}

Although the new game regulation regulates the number of times and the number of guaranteed card drawing, it still has some deficiencies. The main characteristic of gambling is that there is the three aspects of a certain price, dependence on luck and gains. The main reason players spend a lot of money in order to draw cards is to rely on the excitement brought by the uncertainty of luck and the results to achieve the benefits in line with the psychological expectation. Therefore, regulating the number of card draws does not guarantee that players will spend less money. The price required by card draws in today's market is still high in terms of RMB, and 50 is just the time limit of a day, which means players can keep drawing cards for days. So it cannot solve the problem of continuous drawing of addicted players.

Guaranteed probability also does not guarantee that the player will be able to draw the desired props, because there are a variety of types of props in the game, and it is obviously unrealistic to set a guaranteed probability for each prop. If the guaranteed probability of more than ten props is 10 draws, the player cannot win all the props within the range of props guaranteed within 10 draws. Therefore, not every prop can be specified a guarantee probability. In general games, the merchant will classify the props into a level according to their properties, and the props with similar functions and functions in the game will be classified into a category. Taking the mobile game Onmyoji as an example, it divides the props into ssr, sr, $\mathrm{s}$ and other different levels. When the players draw card in the system, the guarantee probability can ensure that the high-level props will be drawn. However, there are many types of medium and high level props, and the guarantee of winning them does not necessarily guarantee a specific one of them. Therefore, this new regulation does not guarantee that players will be able to draw the props they want, but only ensure the fairness of the game to a certain extent. In addition, the 
merchants can also make free riding by listing those obviously not of high level along with the attracting ones with high value on one rank. Even if there is a guarantee probability, they will spare no efforts to make it difficult for the player to draw the desired props, forcing them to spend money to draw cards. Therefore, the guaranteed probability does not completely change the way players spend a lot of money in order to get props.

To sum up, the current restrictions on the card drawing mechanism do not really change the system's reliance on luck and the player's willingness to spend money in order to obtain high-value props.

\section{REGULATION ON THE CARD DRAWING MECHANISM}

\section{A. Suggestions on regulation of card drawing system}

According to the characteristics of gambling, the system is regulated from three aspects: cost, luck and reward.

In terms of cost, although the total number of card draws per day is set at 50 , this does not necessarily reduce the cost of the players. It is necessary to change the limit on the number of card draws per day to the limit on the total amount of money, in case game merchants increase the cost of each card draw because of the time limit. So forcing players to reduce the cost of cards can prevent them from binge spending. It is especially important to increase the restrictions on the juvenile card drawing. Since juvenile's judgment ability is limited and have less moderation than adults, there are always minors who spend a lot of money on a game without their parents knowing about it. Therefore juveniles must be limited in games where draws requires money.

From the aspect of luck, efforts can be made in both the guaranteed draws and the probability, with the focus of the former on the levels of the props and the latter on a specific prop. There shouldn't be any prop of too low probability, in case one of too low probability triggers off over popularity among players who may be stimulated to draw cards. When a prop is too much rare, it might encourage players' curiosity and comparison and thus leads to negative effects. Therefore, in terms of the limitation on luck, not only should there be guaranteed number of times, but there should also be a chance for some rare props to appear. In this way, it is balance between the playability of games and the reason of players, which avoids players from getting a tendency of gamblers.

In the respect of reward, game operators should not advertise too much to stimulate players' psychology, encourage them to draw cards too much, or use too inflammatory words in the game and the community formed by players. When propagandizing props with limit of time and number, they cannot overemphasize the "transience", "out of print" and other words to stimulate the player's desire to draw cards. Players are willing to pay a certain price in order to get the desired reward. The game operators' overemphasis on the rarity and value of props will make the players pursue too much and develop the gambler's mentality.

Therefore, efforts should be made from the three most prominent aspects of gambling to restrict the card drawing system to prevent the system from becoming a disguised form of gambling which produces similar consequences.

\section{B. Not overregulating the card drawing system}

In China, players do not have the habit of paying for the game, the revenue of game operation depends on the players' spending in the game, and card drawing has become a way for businesses to make profits. For the prosperity of the game industry, even though there are similarities between this system and gambling, onesize-fits-all approach should not be adopted in that a complete ban on it does harm to the development of the game industry. In addition, if a merchant encourages spending on draws and set lower probability of rare props, players will have to spend too much money. In this way, the game experience will be negatively influenced, and players will thus gradually drain away. In this case, the game will lose the market under the market selection mechanism. But the regulation on the system cannot be completely relaxed. If the problem is left to the market itself to solve, a bad atmosphere may be formed and bad money will drive out good money. So a certain regulation is required to set up healthy atmosphere and encourage healthy competition among game vendors.

\section{Other problems in the card drawing system}

There can be other problems in the card drawing system, like swindling acquaintances with big data, setting different drop rate for different player. In order to encourage rich players to spend money, the game vendor might rearrange their drop rate in card drawing and make them less likely to win rare props. This problem can be addressed by the "Consumer Protection Act", but it is difficult to collect evidence.

\section{CONCLUSION}

Although the card drawing mechanism has problems such as its similarity to gambling, it should not be completely banned as a fun type of gameplay. It is necessary to draw a good bottom line and take preventive measures to prevent the system from developing towards gambling, promote the healthy development of the electronic game industry, and create a harmonious game environment. 


\section{References}

[1] Ta Ying, Shen Hao, Analysis of the Development Status and Future Trend of Mobile Games [J]. Gansu Science and Technology, 2013, 29(05): 79-80. (in Chinese)

[2] Dong Kun, Mobile Game Development Status and Characteristics [J]. Digital Technology \& Application, 2011(01): 120. (in Chinese)

[3] Liu Junmin, Economics of Virtual Economy [J]. China Opening Herald, 2008(06): 5-11. (in Chinese)

[4] Xi Shenghui, Economic Analysis of Online Game Industry [J]. Shanghai Academy of Social Sciences, 2007. (in Chinese)

[5] Li Gang, An Empirical Analysis of the Status Quo of China's Lottery Industry and Research on Future Development Strategies [J]. Fudan University, 2006. (in Chinese)

[6] Feng Erkang, On the Practice of 'Harming the Familiar' and the Attitudes to the Traditional Culture [J]. Journal of University of Science and Technology of Suzhou: Social Science, 2003(03): 90-93. (in Chinese)

[7] Chen Xia, Huang Xiting, Bai Gang, Psychological Researches on Internet Addiction [J]. Advances In Psychological Science, 2003(03): 355-359. (in Chinese) 